\title{
Composição química e modulação da resistência bacteriana a drogas do óleo essencial das folhas de Rollinia leptopetala R. E. Fries
}

\author{
Vicente C. O. Costa, ${ }^{1}$ Josean F. Tavares, ${ }^{* 1}$ Maria F. Agra, ${ }^{1}$ Vivyanne S. Falcão-Silva, ${ }^{2}$ \\ Roselaine Facanali, ${ }^{3}$ Maria Aparecida R. Vieira, ${ }^{3}$ Márcia Ortiz M. Marques, ${ }^{3}$ José P. \\ Siqueira-Júnior, ${ }^{2}$ Marcelo Sobral da Silva ${ }^{1}$
}

\author{
${ }^{1}$ Laboratório de Tecnologia Farmacêutica, Universidade Federal da Paraíba, Campus I, CP 5009, 58051-970 \\ João Pessoa-PB, Brasil, \\ ${ }^{2}$ Laboratório de Genética de Microrganismos, Departamento de Biologia Molecular, Universidade Federal da \\ Paraiba, 58059-900 João Pessoa-PB, Brasil, \\ ${ }^{3}$ Centro de $P \& D$ de Recursos Genéticos Vegetais, Instituto Agronômico de Campinas, CP 28, \\ 13001-970 Campinas-SP, Brasil
}

\begin{abstract}
RESUMO: O óleo essencial das folhas de Rollinia leptopetala foi obtido por hidrodestilação em aparelho de Clevenger e a sua composição química foi analisada através de CG-EM. Com essa técnica, foi possível identificar 22 constituintes em uma mistura complexa de monoterpenos $(54,5 \%)$ e sesquiterpenos $(45,5 \%)$. O principal componente encontrado nas folhas foi o biciclogermacreno (22,47\%). O óleo essencial foi avaliado numa linhagem de Staphylococcus aureus portadora de bomba de efluxo responsável pela resistência norfloxacino. Embora o óleo essencial não tenha apresentado atividade antibacteriana relevante in vitro, ele apresentou atividade moduladora da resistência, ou seja, em combinação com o norfloxacino observouse uma redução de 4x na concentração inibitória mínima do antibiótico, indicando inibição de bomba de efluxo.
\end{abstract}

Unitermos: Rollinia leptopetala, óleo essencial, CG-EM, Staphylococcus aureus, modulação da resistência a drogas, Inibição de bomba de efluxo.

\begin{abstract}
Chemical composition and modulation of bacterial drug resistance of the essential oil from the leaves of Rollinia leptopetala R. E. Fries". The essential oil from the leaves of Rollinia leptopetala was obtained by hydrodistillation in Clevenger's apparatus and its chemical composition was analyzed by GC-MS. With this technique could be identified 22 constituents in a complex mixture of monoterpenes $(54.5 \%)$ and sesquiterpenes $(45.5 \%)$. The main component found in the leaves was the bicyclogermacrene $(22.47 \%)$. The essential oil was assayed against a strain of Staphylococcus aureus possessing efflux mechanism of resistance to norfloxacin. Although the essential oil did not display relevant antibacterial activity in vitro, it modulated the activity of the norfloxacin, i.e. in combination with the antibiotic it was observed a fourfold reduction in the minimum inhibitory concentration for norfloxacin, indicating inhibition of efflux pump.
\end{abstract}

Keyword: Rollinia leptopetala, essential oil, GC-MS, Staphylococcus aureus, modulation of drug resistance, efflux pump inhibitor.

\section{INTRODUÇÃO}

A família Annonaceae é constituída por cerca de 120 gêneros e aproximadamente 2300 espécies. No Brasil são registrados 29 gêneros, compreendendo cerca de 260 espécies (Barroso, 1978). Para a Paraíba um levantamento das Annonaceae no Herbário JPB, registrou 7 gêneros e 12 espécies (Pontes et al., 2004). Essa família é caracterizada pela presença de terpenóides (principalmente diterpenos), alcalóides (um grande número de derivados do núcleo isoquinolínico), além de óleos essenciais cuja composição química é predominantemente de monoterpenos e sesquiterpenos (Leboeuf et al., 1982). O gênero Rollinia é constituído por 65 espécies, que ocorrem principalmente na América do Sul e com poucas espécies no México, Caribe e América Central (Leboeuf et al., 1982). Existem relativamente poucos relatos de investigações fitoquímicas sobre este gênero comparado com outros, por exemplo, Annona (Kuo et al., 2001; Paulo et al., 1992) e Duguetia (Fechine et al., 2002; Sousa et al., 2004). Apesar de vários trabalhos relatando o isolamento de acetogeninas no gênero Rollinia, existem apenas dois reportando a caracterização de constituintes voláteis (Pino, 2000; 
Jurgens et al., 2000), o que torna esse estudo de alta relevância. Rollinia leptopetala R.E. Fries é uma árvore ou arbusto, endêmica do Brasil com nome popular de "pinha brava" e utilizada pela medicina popular como digestivo (Agra et al., 2007). Trabalhos prévios, realizados por nossa equipe, relataram a presença de alcalóides tetrahidroprotoberberínicos nessa espécie (Sette et al., 2000a,b). Nosso grupo vem trabalhando com constituintes voláteis de espécies de Annonaceae, dentre elas, Xylopia langsdorffiana (Tavares et al., 2007), Fusaea longifolia (Tavares et al., 2005) e Guatteria (Lima et al., 2003; Lima et al., 2004;).

Bombas de efluxo são proteínas integrantes da membrana plasmática bacteriana e que tem sido responsabilizadas por diversos casos de resistência a drogas, as quais são expelidas para fora da célula (Piddock, 2006). Modificadores da atividade antibiótica é um termo usado para substâncias que modulam ou mesmo revertem a resistência bacteriana a certos antibióticos, como é o caso de vários produtos naturais de origem vegetal (extratos e fitoconstituintes) que

Tabela 1. Composição química do óleo essencial das folhas de Rollinia leptopetala.

\begin{tabular}{|c|c|c|}
\hline Composto & Área \% & $\mathrm{IR}^{\mathrm{a}}$ \\
\hline$\alpha$-tujeno & 3,80 & 924 \\
\hline$\alpha$-pineno & 1,80 & 931 \\
\hline sabineno & 0,53 & 968 \\
\hline mirceno & 3,51 & 985 \\
\hline$\delta$-3-careno & 0,57 & 1007 \\
\hline limoneno & 3,74 & 1023 \\
\hline (Z)- $\beta$-ocimeno & 0,70 & 1031 \\
\hline (E)- $\beta$-ocimeno & 2,60 & 1041 \\
\hline isoterpinoleno & 0,69 & 1082 \\
\hline cis-4-tujanol & 17,37 & 1093 \\
\hline$\alpha$-terpineol & 8,42 & 1181 \\
\hline geraniol & 1,79 & 1246 \\
\hline$\beta$-elemeno & 0,52 & 1384 \\
\hline trans-cariofileno & 6,63 & 1410 \\
\hline aromadendreno & 0,68 & 1430 \\
\hline$\alpha$-humuleno & 0,78 & 1444 \\
\hline germacreno D & 7,72 & 1471 \\
\hline biciclogermacreno & 22,47 & 1487 \\
\hline$\delta$-cadineno & 0,83 & 1513 \\
\hline germacreno B & 1,30 & 1545 \\
\hline espatulenol & 0,77 & 1564 \\
\hline guaiol & 4,61 & 1585 \\
\hline
\end{tabular}

a(Índice de Retenção = Índice de Kovats); Componentes identificados baseados no IR e CG-EM e listados de acordo com a ordem de eluição na coluna DB-5 (30 m). alteram a susceptibilidade microbiana a antibióticos por inibição de bombas de efluxo (Gibbons, 2004). No presente trabalho reportamos a composição química do óleo essencial de $R$. leptopetala (OERL) e avaliamos efeito modulador da resistência a drogas usando uma linhagem de Staphylococcus aureus portadora de bomba de efluxo.

\section{MATERIAL E MÉTODOS}

\section{Material vegetal}

O material vegetal (folhas) foi coletado em Serra Branca, região Semi-árida da Paraíba, Brasil, em março de 2005. Uma exsicata está depositada no Herbário Prof. Lauro Pires Xavier (JPB) na Universidade Federal da Paraíba sob identificação Agra 3567.

\section{Extração do óleo essencial}

As folhas frescas de R. leptopetala (1600 g) foram submetidas a hidrodestilação durante $4 \mathrm{~h}$, em aparelho do tipo Clevenger a temperature de $40{ }^{\circ} \mathrm{C}$, obtendo-se $640 \mathrm{mg}$ de óleo essencial. Para análise, o óleo essencial foi diluído da seguinte forma: $2 \mu \mathrm{L}$ de amostra em $1 \mathrm{~mL}$ de acetato de etila.

\section{Análise do óleo essencial}

A análise em $\mathrm{CG}$ foi realizada em um cromatógrafo Shimadzu GC17-A, usando coluna capilar de sílica fundida DB-5 (30 m x 0,25 mm d.i., 0,25 $\mu \mathrm{M}$ de espessura do filme). Foi utilizado Hélio como gás carreador, a um fluxo de $1,0 \mathrm{~mL} / \mathrm{min}$. A temperatura do forno foi programada de $60^{\circ}$ a $240^{\circ}$ a $3{ }^{\circ} \mathrm{C} / \mathrm{min}$. As temperaturas do injetor e do detector foram de $220^{\circ} \mathrm{C}$ e $230{ }^{\circ} \mathrm{C}$, respectivamente.

A análise por Cromatografia a Gás Espectrometria de Massas (CG-EM) foi realizada em um sistema Shimadzu QP-5000-Quadrupole MS, operando com energia de ionização de $70 \mathrm{eV}$. Foi utilizada coluna capilar de sílica fundida DB-5 (30 m x $0,25 \mathrm{~mm}$ d.i., $0,25 \mu \mathrm{M}$ de espessura do filme); Hélio como gás carreador, fluxo de $1 \mathrm{~mL} / \mathrm{min}$ com split. As temperaturas do injetor e do detector foram de $220{ }^{\circ} \mathrm{C}$ e $230{ }^{\circ} \mathrm{C}$, respectivamente. A temperatura da coluna foi programada de $60^{\circ} \mathrm{C}$ a $240{ }^{\circ} \mathrm{C}$ a $3{ }^{\circ} \mathrm{C} / \mathrm{min}$. A identificação das substâncias foi efetuada através da comparação dos seus espectros de massas com o banco de dados do sistema CG-EM (Nist. 62 lib.) e índice de retenção de Kovats (Adams, R. P. Identification of essential oil components by cromatography / mass spectrocopy. Allured Publ. Corp., Carol Stream, 1995). Os índices de retenção das substâncias foram obtidos pela coinjeção do óleo essencial com uma mistura padrão de hidrocarbonetos $\left(\mathrm{C}_{9}-\mathrm{C}_{24}\right)$, aplicando-se a equação de Van den Dool \& Kratz, 1963. 


\section{Ensaios bacteriológicos}

A linhagem de $S$. aureus utilizada foi a SA1199B que superexpressa o gene nor $A$ codificador da proteína NorA de efluxo do norfloxacino e outras drogas (Kaatz et al., 1993; Kaatz \& Seo, 1995). A solução estoque do norfloxacino (Sigma-Aldrich) foi preparada em água após solubilização com $\mathrm{NaOH} \mathrm{0,1} \mathrm{M} \mathrm{(CLSI/}$ NCCLS document M100-S15). A solução estoque do óleo essencial foi preparada em Tween-80 (SigmaAldrich). A concentração inibitória mínima (CIM) do norfloxacino e do OERL foi determinada pelo método da diluição em meio de cultura solidificado (agar infusão de coração, Difco) com concentrações variando de 0,25 a $128 \mu \mathrm{g} / \mathrm{mL}$ e 0,03125 a $2 \%$, respectivamente. A CIM foi definida como a menor concentração que inibia completamente o crescimento bacteriano. Para a avaliação do OERL como modulador da resistência, a CIM do norfloxacino foi determinada na presença do óleo numa concentração subinibitória.

\section{RESULTADOS E DISCUSSÃO}

O óleo essencial das folhas foi obtido por hidrodestilação com rendimento de $0,04 \%$ em relação ao peso de material fresco utilizado. Pela análise em CG-EM foi possível identificar 22 componentes, correspondendo a 91,83\% do total do óleo em uma mistura complexa de monoterpenos (54,5\%) e sesquiterpenos (45,5\%). Biciclogermacreno $(22,47$ $\%$, cis-4-tujanol (17,37 \%), $\alpha$-terpineol (8,42 \%), germacreno D (7,72\%), trans-cariofileno $(6,63 \%)$ e guaiol $(4,61 \%)$ foram os constituintes majoritários. Essa composição química é compatível com dados da literatura para constituintes voláteis de espécies de Rollinia (Pino, 2000; Jurgens et al., 2000) bem como, de outras espécies de Annonaceae cuja composição dos componentes voláteis é caracteristicamente de monoterpenos e sesquiterpenos (Tavares et al., 2007). A Tabela 1 mostra os componentes do óleo essencial, bem como suas porcentagens e Índice de Kovats.

$\mathrm{Na}$ ausência do OERL no meio de cultura, a CIM do norfloxacino observada foi $64 \mu \mathrm{g} / \mathrm{mL}$. Embora o OERL não tenha apresentado atividade antibacteriana relevante $(\mathrm{CIM}=0,5 \%)$, quando foi incorporado ao meio de cultura na concentração $0,0625 \%$ (1/8 CIM), a CIM do norfloxacino observada foi $16 \mu \mathrm{g} / \mathrm{mL}$ (redução de 4X), indicando inibição da bomba de efluxo.

É provocativa e lícita a idéia de que os componentes principais do OERL, biciclogermacreno e cis-4-tujanol, sejam os responsáveis pela observada atividade moduladora da resistência. Experimentos estão planejados para avaliar tal idéia.

Alguns poucos trabalhos relatam o efeito potencializador de óleos essenciais quando em combinação com drogas antimicrobianas clássicas (Filoche et al., 2005; Shahverdi et al., 2007; Rosato et al., 2007; Oliveira et al., 2006; Oliveira et al., 2007), mas digno de nota mesmo é o fato de termos encontrado na literatura apenas um artigo avaliando óleo essencial (Ligustica porteri L, Apiaceae) como modulador de resistência a drogas por inibição de bomba de efluxo (Cégiéla-Carlioz et al., 2005), sendo bom aqui mencionar que o OERL apresentou melhor atividade moduladora.

Destarte, os resultados aqui apresentados mostram a relevância da avaliação de óleos essenciais de espécies vegetais nativas como inibidores putativos de bomba de efluxo, ou seja, potenciais adjuvantes de antibióticos.

\section{AGRADECIMENTOS}

Dr. Simon Gibbons (Universidade de Londres) pela valiosa e gentil cooperação, as agências de fomento: CNPq, CAPES, FAPESQ-PB, RENORBIO e IMSEAR e a Raimundo Nonato da Silva Filho pelo apoio técnico.

\section{REFERÊNCIAS}

Agra MF, Freitas PF, Barbosa-Filho JM 2007. Synopsis of the plants known as medicinal and poisonous in Northeast of Brazil. Rev Bras Farmacogn 17: 114-140.

Barroso GM 1978. Sistemática de angiospermas do Brasil. São Paulo: Ed. da Universidade de São Paulo.

Cégiéla-Carlioz P, Bessière J, David B, Mariotte A, Gibbons S, Dijoux-Franca M 2005. Modulation of multidrug resistance (MDR) in Staphylococcus aureus by Osha (Ligusticum porteri L., Apiaceae) essential oil compounds. Flavour Frag J. 20: 671-675.

Fechine IM, Navarro VR, Cunha EVL, Silva MS, Maia JGS, Barbosa-Filho JM 2002. Alkaloids and volatile constituents from Duguetia flagellaris. Biochem Syst Ecol 30: 267-269.

Filoche SK, Soma K, Sissons CH 2005. Antimicrobial effects of essential oils in combination with chlorhexidine digluconate. Oral Microbiol Immunol 20: 221-225.

Gibbons S 2004. Anti-staphylococcal plant natural products. Nat Prod Reps 21: 263-277.

Jurgens A, Webber AC, Gottsberger G 2000. Floral scent compounds of Amazoniam Annonaceae species pollinated by small beetles and thrips. Phytochemistry 55: 551-558.

Kaatz GW, Seo SM, Ruble CA 1993. Efflux-mediated fluoroquinolone resistance in Staphylococcus aureus. Antimicrob Agents Chemother 37: 1086-1094.

Kaatz GW, Seo SM 1995. Inducible NorA-mediated multidrug resistance in Staphylococcus aureus. Antimicrob Agents Chemother 39: 2650-2655.

Kuo RY, Chang FR, Chen CY, Teng CM, Yen HF, Wu YC 2001. Antiplatelet activity of $N$-methoxycarbonyl aporphines from Rollinia mucosa. Phytochemistry 57: 421-425.

Lebouef M, Cavé A, Bhaumik PK, Mukerjee B, Mukherjee R 1982. The phytochemistry of the Annonaceae. Phytochemistry 21: 2783-2813.

Lima MA, Fechine IM, Silva MS, Maia JGS, Cunha EVL, Barbosa-Filho JM 2003. Alkaloids and volatile constituents from Guatteria juruensis. Biochem Syst 
Ecol 31: 423-425.

Lima MA, Barbosa-Filho JM, Maia JGS, Silva MS, Cunha EVL 2004. Alkaloids and volatile constituents from Guatteria poeppigiana. Biochem Syst Ecol 32: 347-349.

Oliveira RAG, Lima EO, Vieira WL, Freire KRL, Trajano VN, Lima, IO, Souza EL, Toledo MS, Silva-Filho RN 2006. Estudo da interferência de óleos essenciais sobre a atividade de alguns antibióticos usados na clínica. Rev Bras Farmacogn 16: 77-82.

Oliveira RAG, Lima EO, Souza EL, Vieira WL, Freire KRL, Trajano VN, Lima IO, Silva-Filho RN 2007. Interference of Plectranthus amboinicus (Lour.) Spreng essential oil on the anti-Candida activity of some clinically used antifungals. Rev Bras Farmacogn 17: 186-190.

Paulo MQ, Barbosa-Filho JM, Lima EO, Maia RF, Barbosa RCSBC, Kaplan MAC 1992. Antimicrobial activity of benzylisoquinoline alkaloids from Annona salzmanii D.C. J Ethnopharmacol 36: 39-41.

Piddock LJV 2006. Clinically relevant chromosomally encoded multidrug resistance efflux pumps in bacterial. Clin Microbiol Rev 19: 382-402.

Pino JA 2000. Volatile components of Rollinia mucosa (Jacq.) Baill. J Essent Oil Res 12: 97-98.

Pontes AF, Barbosa MRV, Maas PJM 2004. Flora Paraibana: Annonaceae Juss. Acta Bot Bras 18: 281-293.

Rosato A, Vitali C, De Laurentis N, Armenise D, Milillo MA 2007. Antibacterial effect of some essential oil administered alone or in combination with norfloxacin. Phytomedicine 14: 727-732.

Sette IMF, Da-Cunha EVL, Barbosa-Filho JM, Agra MF, DaSilva MS 2000a. The first tetrahydroprotoberberine alkaloid from the genus Rollinia. Biochem Syst Ecol 28: 393-394.

Sette IMF, Cunha EVL, Barbosa-Filho JM, Silva MS 2000 b. Tetrahydroprotoberberine and aporphine alkaloids from Rollinia leptopetala. Pharm Biol 38: 318-320.

Shahverdi AR, Abdolpour F, Monsef-Esfahani HR, Farsan H 2007. A TLC bioautographic assay for the detection of nitrofurantoin resistance reversal compound. $J$ Chromatogr B 850: 528-530.

Sousa OV, Soares-Júnior DT, Del-Vechio G, Mattosinhos RG, Gattas CR, Kaplan MAC 2004. Atividades antinociceptiva e antiinflamatória do óleo essencial de cascas de Duguetia lanceolata St. Hil., Annonaceae. Rev Bras Farmacogn 14 (Supl): 11-14.

Tavares JF, Barbosa-Filho JM, Da-Silva MS, Maia JGS, DaCunha EVL 2005. Alkaloids and volatile constituents from the stem of Fusaea longifolia (Aubl.) Saff. (Annonaceae). Rev Bras Farmacogn 15: 115-118.

Tavares JF, Silva MVB, Queiroga KF, Martins RM, Silva TMS, Camara CA, Agra MF, Barbosa-Filho JM, Da-Silva MS 2007. Composition and molluscicidal properties of essential oils from leaves of Xylopia langsdorffiana A. St. Hil et Tul. (Annonaceae). $J$ Essent Oil Res 19: 282-284. 